\title{
ITÔ-SKOROHOD STOCHASTIC EQUATIONS AND APPLICATIONS TO FINANCE
}

\author{
CIPRIAN A. TUDOR
}

Received 6 November 2003 and in revised form 8 June 2004

We prove an existence and uniqueness theorem for a class of Itô-Skorohod stochastic equations. As an application, we introduce a Black-Scholes market model where the price of the risky asset follows a nonadapted equation.

\section{Introduction}

The introduction of the anticipating (or Skorohod) integral in [8] and of the anticipating stochastic calculus in [7] has opened the question of solving anticipating stochastic differential equations. In general, the existence and uniqueness of the solution for these equations is not known. The difficulty of solving such equations is due to the fact that the classical method of Picard iterations cannot be applied because the mean square formula for the Skorohod integral involves the Malliavin derivation in a such way that we cannot find "closed" formulas. Only in few particular cases do some results exist; see, for example, $[1,2,3]$. We have recently proved in [9] that the set of Skorohod integrals coincides with a set of integrals of Itô type. In the present work, using this correspondence between Skorohod integrals and Itô-Skorohod integrals, we introduce a class of anticipating equations (called Itô-Skorohod equations) that can be solved using standard techniques. As an application, we introduce a market model where the price of the risky asset follows such an equation with a random initial condition (the price at the transaction time). We prove that our model is complete and has no arbitrage opportunities and we derive a BlackScholes formula when the initial price of the risky asset is given by a standard normal random variable.

We organized the paper as follows. Section 2 contains some preliminaries on the anticipating stochastic calculus. In Section 3, we define the class of Itô-Skorohod equations and we prove the existence and uniqueness of the solution. In Section 4, we introduce a market model with price dynamics following an Itô-Skorohod equation and we obtain a Black-Scholes option valuation formula and the expression of the replicant portfolio. 


\section{Preliminaries}

We start with some elements of the Malliavin calculus. We refer to [6] for a complete presentation of this topic. Let $(W(t))_{t \in[0,1]}$ be a standard Wiener process on the canonical Wiener space $(\Omega, \mathbb{F}, P)$ and let $\left(\mathbb{F}_{t}\right)_{t \in[0,1]}$ be the filtration generated by $W$. A functional of the Brownian motion of the form

$$
F=f\left(W\left(t_{1}\right), \ldots, W\left(t_{n}\right)\right)
$$

with $t_{1}, \ldots, t_{n} \in[0,1]$ and $f \in C_{b}^{\infty}\left(\mathbb{R}^{n}\right)$, is called a smooth random variable and this class is denoted by $\mathscr{Y}$. The Malliavin derivative is defined on $\mathscr{Y}$ as

$$
D_{t} F=\sum_{i=1}^{n} \frac{\partial f}{\partial x_{i}}\left(W\left(t_{1}\right), \ldots, W\left(t_{n}\right)\right) 1_{\left[0, t_{i}\right]}(t), \quad t \in[0,1]
$$

if $F$ has the form (2.1). The operator $D$ is closable and can be extended to the closure of $\mathscr{S}$ with respect to the seminorm

$$
\|F\|_{k, p}^{p}=\mathbb{E}|F|^{p}+\sum_{j=1}^{k} \mathbb{E}\left\|D^{(j)} F\right\|_{L^{2}([0,1])}^{p}
$$

where $D^{(i)}$ denotes the $i$ th iterated derivative. The adjoint of $D$ is denoted by $\delta$ and is called the Skorohod integral. That is, $\delta$ is defined on its domain

$$
\operatorname{Dom}(\delta)=\left\{u \in L^{2}([0,1] \times \Omega) /\left|\mathbb{E} \int_{0}^{1} u_{s} D_{s} F d s\right| \leq C\|F\|_{L^{2}(\Omega)}\right\}
$$

and is given by the duality relationship

$$
\mathbb{E}(F \delta(u))=\mathbb{E} \int_{0}^{1} u_{s} D_{s} F d s, \quad u \in \operatorname{Dom}(\delta), F \in \mathscr{Y} .
$$

Recall that the variance of the Skorohod integral is

$$
\mathbb{E}\left(\delta^{2}(u)\right)=\mathbb{E} \int_{0}^{1} u_{\alpha}^{2} d \alpha+\mathbb{E} \int_{0}^{1} \int_{0}^{1} D_{\beta} u_{\alpha} D_{\alpha} u_{\beta} d \alpha d \beta
$$

By $\mathbb{L}^{k, p}$, we denote the set $L^{2}\left([0,1] ; \mathbb{D}^{k, p}\right)$, for $k \geq 1$ and $p \geq 2$, and we note that $\mathbb{L}^{k, p}$ is a subset of the domain of $\delta$. The following version of the Ocone-Clark formula was given in [7]:

$$
F=\mathbb{E}\left(F / \mathbb{F}_{[s, t]^{c}}\right)+\int_{s}^{t} \mathbb{E}\left(D_{\alpha} F / \mathbb{F}_{[\alpha, t]^{c}}\right) d W(\alpha), \quad \text { for } F \in \mathbb{D}^{1,2}
$$


We will need the integration-by-parts formula

$$
F \delta(u)=\delta(F u)+\int_{[0,1]} D_{s} F u_{s} d s
$$

if all above terms are defined. Recall also that if $F$ is a random variable, Malliavin differentiable, and measurable with respect to a $\sigma$-algebra $\mathbb{F}_{A}, A \in \mathscr{B}(\mathbb{R})$, then

$$
D F=0, \quad \text { on } A^{c} \times \Omega .
$$

We define, for $k \geq 1$ and $p \geq 2$, the sets of processes

$$
\begin{aligned}
& \mathcal{M}^{k, p}=\left\{X=\left(X_{t}\right)_{t \in[0,1]}, X_{t}=\int_{0}^{t} u_{s} d W_{s}, u \in \mathbb{L}^{k, p}\right\} \\
& \mathcal{N}^{k, p}=\left\{Y=\left(Y_{t}\right)_{t \in[0,1]}, Y_{t}=\int_{0}^{t} \mathbb{E}\left[v_{s} / \mathbb{F}_{[s, t]^{c}}\right] d W_{s}, v \in \mathbb{L}^{k, p}\right\} .
\end{aligned}
$$

We will refer to the elements of $\mathcal{N}^{k, p}$ as Itô-Skorohod integral processes and to the elements of $\mathcal{M}^{k, p}$ as Skorohod integral processes. It has been proved in [9] that for sufficiently regular integrands, the two classes coincide. As a consequence, to study Skorohod integral processes, it suffices to study Itô-Skorohod integral processes, which have two interesting properties. Firstly, note that the integral $Y_{t}=\int_{0}^{t} \mathbb{E}\left[u_{\alpha} / \mathbb{F}_{[\alpha, t] c}\right] d W_{\alpha}$ exists even for $u \in L^{2}([0,1] \times \Omega)$ and has similarities to a classical Itô integral. Observe, by (2.6), that this integral is an "isometry":

$$
\mathbb{E}\left(\int_{0}^{t} \mathbb{E}\left[u_{\alpha} / \mathbb{F}_{[\alpha, t]^{c}}\right] d W_{\alpha}\right)^{2}=\mathbb{E} \int_{0}^{t}\left(\mathbb{E}\left[u_{\alpha} / \mathbb{F}_{[\alpha, t]^{c}}\right]\right)^{2} d \alpha
$$

Secondly, if we define, for every $\lambda \leq t, Y_{t}^{\lambda}=\int_{0}^{\lambda} \mathbb{E}\left[u_{\alpha} / \mathbb{F}_{[\alpha, t]^{c}}\right] d W_{\alpha}$, then the process $\left(Y_{t}^{\lambda}\right)_{\lambda \leq t}$ is an $\mathbb{F}_{(\lambda, t]^{c}}$-martingale and we have

$$
\lim _{\lambda \rightarrow t, \lambda \leq t} Y_{t}^{\lambda}=Y_{t} \quad \text { a.s. and in } L^{2} .
$$

We will now define the stochastic integral with respect to Itô-Skorohod integral processes. Definition 2.1. Let $u, v \in L^{2}([0,1] \times \Omega)$ be adapted processes and consider more $Y_{t}=Y_{0}+$ $\int_{0}^{t} \mathbb{E}\left[u_{\alpha} / \mathbb{F}_{[\alpha, t] c}\right] d W_{\alpha}+\int_{0}^{t} \mathbb{E}\left[v_{\alpha} / \mathbb{F}_{[\alpha, t] c}\right] d \alpha$. By definition, for any adapted square integrable process $X$,

$$
\int_{0}^{t} X_{s} d Y_{s}:=\int_{0}^{t} X_{s} d_{s} Y_{t}^{s}
$$

where

$$
Y_{t}^{\lambda}=Y_{0}+\int_{0}^{\lambda} \mathbb{E}\left[u_{\alpha} / \mathbb{F}_{[\alpha, t] c}\right] d W_{\alpha}+\int_{0}^{\lambda} \mathbb{E}\left[v_{\alpha} / \mathbb{F}_{[\alpha, t] c}\right] d \alpha
$$

and the integral on the right-hand side of (2.13) is understood in the semimartingale sense. 


\section{Itô-Skorohod stochastic equations}

In this section, we state and prove an existence and uniqueness theorem for a class of anticipating stochastic differential equations using the method of Picard iterations. It is known that in the anticipating stochastic calculus, this method cannot be applied because the formula of the mean square of the Skorohod integral involves the Malliavin derivative and one cannot find "closed" formulas. We define here a new class of anticipating equations, located "between" Itô and Skorohod equations, that can be solved by classical techniques. Consider the following stochastic differential equation:

$$
X_{t}=Z+\int_{0}^{t} \sigma\left(s, \mathbb{E}\left(X_{s} / \mathbb{F}_{[s, t] c}\right)\right) d W_{s}+\int_{0}^{t} b\left(s, X_{s}\right) d s
$$

Note that the stochastic integral above is a Skorohod integral since the integrand is not adapted and the initial condition is anticipating. The solution will also be anticipating. In what follows, the coefficients $\sigma(t, x), b(t, x):[0,1] \times \mathbb{R} \rightarrow \mathbb{R}$ are given and satisfy the following standard conditions.

(H1) (Measurability): $\sigma$ and $b$ are jointly measurable in $(t, x) \in[0,1] \times \mathbb{R}$.

(H2) (Lipschitz condition): there exists a $D>0$ such that for all $t \in[0,1]$ and $x \in \mathbb{R}$,

$$
|\sigma(t, x)-\sigma(t, y)|+|b(t, x)-b(t, y)| \leq D|x-y|
$$

(H3) (Linear growth condition): there exists a $C>0$ such that for all $t \in[0,1]$ and $x \in \mathbb{R}$

$$
|\sigma(t, x)|^{2}+|b(t, x)|^{2} \leq C^{2}\left(1+|x|^{2}\right) .
$$

We also make a hypothesis concerning the initial value $Z$.

(H4) $Z$ is a random variable with $\mathbb{E}|Z|^{2}<\infty$.

A square integrable process that satisfies a.s. (3.1) is called a solution of (3.1). For given coefficients $\sigma$ and $b$, any solution $X$ will depend on the initial value $Z$. We will say that the solution is unique if, for every $t \in[0,1], P\left(X_{t}^{1}=X_{t}^{2}\right)=1$ for any two solutions $X^{1}$ and $X^{2}$ with the same initial condition.

We start by proving the existence and the uniqueness of the solution of (3.1).

Theorem 3.1. Under assumptions (H1), (H2), (H3), and (H4), stochastic equation (3.1) has a unique solution $X_{t}$ on $[0,1]$ with

$$
\sup _{0 \leq t \leq 1} \mathbb{E}\left|X_{t}\right|^{2}<\infty
$$

Proof. Throughout this proof, $K$ will denote a generic constant depending only on $D$ and $\mathbb{E}|Z|^{2}$. We consider the usual Picard iterations $X_{t}^{(0)}=Z$ and

$$
X_{t}^{(n+1)}=Z+\int_{0}^{t} \sigma\left(s, \mathbb{E}\left(X_{s}^{(n)} / \mathbb{F}_{[s, t] c}\right)\right) d W_{s}+\int_{0}^{t} b\left(s, X_{s}^{(n)}\right) d s
$$


We first prove the existence of the solution. We have, from (2.6), (H3), and Hölder's inequalities, that

$$
\begin{aligned}
\mathbb{E}\left|X_{t}^{(1)}-X_{t}^{(0)}\right|^{2} & \leq 2 \mathbb{E}\left|\int_{0}^{t} \sigma\left(s, \mathbb{E}\left(Z / \mathbb{E}_{[s, t] c}\right)\right) d W_{s}\right|^{2}+2 \mathbb{E}\left|\int_{0}^{t} b(s, Z) d s\right|^{2} \\
& \leq 2 \mathbb{E} \int_{0}^{t}\left|\sigma\left(s, \mathbb{E}\left(Z / \mathbb{E}_{[s, t] c}\right)\right)\right|^{2} d s+2 t \mathbb{E} \int_{0}^{t}|b(s, Z)|^{2} d s \\
& \leq 2 C^{2} \mathbb{E} \int_{0}^{t}\left(1+\left|\mathbb{E}\left(Z / \mathbb{F}_{[s, t] c}\right)\right|^{2}\right) d s+2 t C^{2} \mathbb{E} \int_{0}^{t}\left(1+|Z|^{2}\right) d s \\
& \leq K t .
\end{aligned}
$$

Using the same arguments and condition (H4), we obtain

$$
\begin{aligned}
\mathbb{E}\left|X_{t}^{(n+1)}-X_{t}^{(n)}\right|^{2} \leq & 2 \mathbb{E}\left|\int_{0}^{t}\left(\sigma\left(s, \mathbb{E}\left(X_{s}^{(n)} / \mathbb{F}_{[s, t] c}\right)\right)-\sigma\left(s, \mathbb{E}\left(X_{s}^{(n-1)} / \mathbb{F}_{[s, t] c}\right)\right)\right) d W_{s}\right|^{2} \\
& +2 \mathbb{E}\left|\int_{0}^{t}\left(b\left(s, X_{s}^{(n)}\right)-b\left(s, X_{s}^{(n-1)}\right)\right) d s\right|^{2} \\
\leq & 2 \mathbb{E} \int_{0}^{t}\left(\sigma\left(s, \mathbb{E}\left(X_{s}^{(n)} / \mathbb{F}_{[s, t] c}\right)\right)-\sigma\left(s, \mathbb{E}\left(X_{s}^{(n-1)} / \mathbb{F}_{[s, t] c}\right)\right)\right)^{2} d s \\
& +2 t \mathbb{E} \int_{0}^{t}\left(b\left(s, X_{s}^{(n)}\right)-b\left(s, X_{s}^{(n-1)}\right)\right)^{2} d s \\
\leq & 2 D^{2}(1+t) \int_{0}^{t} \mathbb{E}\left|X_{s}^{(n)}-X_{s}^{(n-1)}\right|^{2} d s .
\end{aligned}
$$

By induction, one can show that there exists $K>0$ such that for all $t \in[0,1]$ and $n \geq 1$,

$$
\mathbb{E}\left|X_{t}^{(n+1)}-X_{t}^{(n)}\right|^{2} \leq \frac{(K t)^{n+1}}{(n+1) !}
$$

Relation (3.8) and standard arguments imply the convergence, in $L^{2}(\Omega)$, of the successive approximations $X_{t}^{(n)}$ to a limit $X_{t}$ defined by $X_{t}=Z+\sum_{n=0}^{\infty}\left(X_{t}^{(n+1)}-X_{t}^{(n)}\right)$.

To prove that $X$ is a solution, we take the $L^{2}(\Omega)$-limit in (3.5) as $n \rightarrow \infty$. Obviously,

$$
\begin{aligned}
& 2 \mathbb{E}\left|\int_{0}^{t}\left(\sigma\left(s, \mathbb{E}\left(X_{s}^{(n)} / \mathbb{F}_{[s, t]^{c}}\right)\right)-\sigma\left(s, \mathbb{E}\left(X_{s} / \mathbb{F}_{[s, t] c}\right)\right)\right) d W_{s}\right|^{2} \\
& \quad \leq K \int_{0}^{t} \mathbb{E}\left|X_{s}^{(n)}-X_{s}\right|^{2} d s \longrightarrow_{n \rightarrow \infty} 0, \\
& \mathbb{E}\left|\int_{0}^{t}\left(b\left(s, X_{s}^{(n)}\right)-b\left(s, X_{s}\right)\right) d s\right|^{2} \leq K \int_{0}^{t} \mathbb{E}\left|X_{s}^{(n)}-X_{s}\right|^{2} d s \longrightarrow_{n \rightarrow \infty} 0 .
\end{aligned}
$$


The uniqueness of the solution is given by Gronwall's lemma since for any two solutions $X, Y$ with the same initial condition and for every $t \in[0,1]$, we have

$$
\mathbb{E}\left|X_{t}-Y_{t}\right|^{2} \leq K \int_{0}^{t} \mathbb{E}\left|X_{s}-Y_{s}\right|^{2} d s
$$

Concerning bound (3.4), we will only note that standard techniques apply (see, e.g., [4]).

Remark 3.2. We define the following stochastic differential equation:

$$
X_{t}=\mathbb{E}\left(Z / \mathbb{F}_{t^{c}}\right)+\int_{0}^{t} \sigma\left(s, \mathbb{E}\left(X_{s} / \mathbb{F}_{[s, t]^{c}}\right)\right) d W_{s}+\int_{0}^{t} b\left(s, \mathbb{E}\left(X_{s} / \mathbb{F}_{[s, t]^{c}}\right)\right) d s .
$$

Following the lines of the proof of Theorem 3.1, one can show that (3.11) admits a unique solution $X$ with $\sup _{0 \leq t \leq 1} \mathbb{E}\left|X_{t}\right|^{2}<\infty$.

In the particular case of linear coefficients, one can explicitly obtain the solution of (3.11).

Corollary 3.3. Let $\sigma, b \in \mathbb{R}$ and $X_{0} \in L^{2}(\Omega)$. Consider the equation

$$
X_{t}=\mathbb{E}\left(X_{0} / \mathbb{F}_{t^{c}}\right)+\int_{0}^{t} \sigma \mathbb{E}\left(X_{s} / \mathbb{F}_{[s, t]^{c}}\right) d W_{s}+\int_{0}^{t} b \mathbb{E}\left(X_{s} / \mathbb{F}_{[s, t]^{c}}\right) d s .
$$

Then the unique solution of (3.12) is given by

$$
X_{t}=\mathbb{E}\left(X_{0} / \mathbb{F}_{t^{c}}\right) e^{\sigma W_{t}+\left(b-\sigma^{2} / 2\right) t} .
$$

Proof. Denote $M_{t}=e^{\sigma W_{t}+\left(b-\sigma^{2} / 2\right) t}$. Then $M_{t}$ satisfies the equation

$$
M_{t}=1+\int_{0}^{t} \sigma M_{s} d W_{s}+\int_{0}^{t} b M_{s} d s
$$

and using (2.8) and (2.9), we obtain

$$
\begin{aligned}
X_{t} & =\mathbb{E}\left(X_{0} / \mathbb{F}_{t^{c}}\right) M_{t} \\
& =\mathbb{E}\left(X_{0} / \mathbb{F}_{t^{c}}\right)+\int_{0}^{t} \sigma \mathbb{E}\left(X_{0} / \mathbb{F}_{t^{c}}\right) M_{s} d W_{s}+\int_{0}^{t} b \mathbb{E}\left(X_{0} / \mathbb{F}_{t^{c}}\right) M_{s} d s \\
& =\mathbb{E}\left(X_{0} / \mathbb{F}_{[s, t]^{c}}\right)+\int_{0}^{t} \sigma \mathbb{E}\left(\mathbb{E}\left(X_{0} / \mathbb{F}_{s^{c}}\right) M_{s} / \mathbb{F}_{[s, t]^{c}}\right) d W_{s}+\int_{0}^{t} b \mathbb{E}\left(\mathbb{E}\left(X_{0} / \mathbb{F}_{s^{c}}\right) M_{s} / \mathbb{F}_{[s, t]^{c}}\right) d s \\
& =\mathbb{E}\left(X_{0} / \mathbb{F}_{t^{c}}\right)+\int_{0}^{t} \sigma \mathbb{E}\left(X_{s} / \mathbb{F}_{[s, t]^{c}}\right) d W_{s}+\int_{0}^{t} b \mathbb{E}\left(X_{s} / \mathbb{F}_{[s, t]^{c}}\right) d s .
\end{aligned}
$$

\section{Black-Scholes model driven by Itô-Skorohod stochastic differential equations}

We introduce, in this section, a market model with price dynamics following an ItôSkorohod stochastic equation. As usual, we will consider two assets on the probability 
space $\left(\Omega, \mathbb{F}, P,\left(\mathbb{F}_{t}\right)_{t \in[0,1]}\right)$ : the safe investment $A=\left(A_{t}\right)_{t \in[0,1]}$ satisfying $A_{t}=1+r \int_{0}^{t} A_{s} d s$ and the risky asset $S=\left(S_{t}\right)_{t \in[0,1]}$ with price dynamics following the stochastic differential equation

$$
S_{t}=\mathbb{E}\left(S_{0} / \mathbb{F}_{t^{c}}\right)+\int_{0}^{t} \sigma \mathbb{E}\left(S_{s} / \mathbb{F}_{[s, t]^{c}}\right) d W_{s}+\int_{0}^{t} b \mathbb{E}\left(S_{s} / \mathbb{F}_{[s, t]^{c}}\right) d s .
$$

Clearly, $A_{t}=e^{r t}$ and Corollary 3.3 implies that

$$
S_{t}=\mathbb{E}\left(S_{0} / \mathbb{F}_{t^{c}}\right) e^{\sigma W_{t}+\left(b-\sigma^{2} / 2\right) t}
$$

The value of the portfolio at the instant $t$ is defined by

$$
V_{t}=h_{t} A_{t}+H_{t} S_{t}
$$

where the components $h, H \in L^{2}([0,1] \times \Omega)$ are adapted to the Brownian filtration and represent the quantities of the safe asset and the risky asset at the instant $t$.

We say that the portfolio $\left(h_{t}, H_{t}\right)_{t \in[0,1]}$ is self-financing if

$$
V_{t}=\mathbb{E}\left(V_{0} / \mathbb{F}_{t^{c}}\right)+\int_{0}^{t} h_{s} d A_{s}+\int_{0}^{t} H_{s} d S_{s}
$$

where the differential $d S$ is understood in the sense of Definition 2.1.

Remark 4.1. Note that Definition 2.1 can be used although the initial value depends on $t$ because, by the Ocone-Clark formula (2.7), we can write

$$
\mathbb{E}\left(S_{0} / \mathbb{F}_{t^{c}}\right)=S_{0}-\int_{0}^{t} \mathbb{E}\left(D_{s} S_{0} / \mathbb{F}_{[s, t] c}\right) d W_{s}
$$

In other words, the self-financing condition (4.4) can be written as

$$
\begin{aligned}
V_{t}= & \mathbb{E}\left(V_{0} / \mathbb{F}_{t^{c}}\right)+\int_{0}^{t} h_{s} r e^{r s} d s-\int_{0}^{t} H_{s} \mathbb{E}\left(D_{s} S_{0} / \mathbb{E}_{[s, t]^{c}}\right) d W_{s} \\
& +\int_{0}^{t} H_{s} \sigma \mathbb{E}\left(S_{s} / \mathbb{F}_{[s, t]^{c}}\right) d W_{s}+\int_{0}^{t} b H_{s} \mathbb{E}_{\left(S_{s} / \mathbb{F}_{[s, t]^{c}}\right) d s .}
\end{aligned}
$$

In the following, we will denote by $\tilde{S}_{t}=e^{-r t} S_{t}$ the discounted risky asset price. A necessary and sufficient condition for the portfolio, to be self-financing, is given in the next result.

Proposition 4.2. Assume that $h, H \in L^{2}([0,1] \times \Omega)$ and let the process $V$ be given by (4.3). Denote $\tilde{V}_{t}=e^{-r t} V_{t}$. Then the portfolio is self-financing if and only if

$$
\tilde{V}_{t}=\mathbb{E}\left(V_{0} / \mathbb{F}_{t^{c}}\right)+\int_{0}^{t} H_{s} d \tilde{S}_{s} \quad \text { for every } t \in[0,1]
$$


Proof. Suppose that $V$ satisfies (4.4). Define, for every $\lambda \in[0, t]$,

$$
\begin{aligned}
V_{\lambda, t}= & \mathbb{E}\left(V_{0} / \mathbb{F}_{t^{c}}\right)+\int_{0}^{\lambda} h_{s} r e^{r s} d s-\int_{0}^{\lambda} H_{s} \mathbb{E}\left(D_{s} S_{0} / \mathbb{F}_{[s, t]^{c}}\right) d W_{s} \\
& +\int_{0}^{\lambda} H_{s} \sigma \mathbb{E}\left(S_{s} / \mathbb{F}_{[s, t]^{c}}\right) d W_{s}+\int_{0}^{\lambda} b H_{s} \mathbb{E}\left(S_{s} / \mathbb{F}_{[s, t]^{c}}\right) d s .
\end{aligned}
$$

It is not difficult to check that $V_{\lambda, t}=\mathbb{E}\left(V_{\lambda} / \mathbb{F}_{[\lambda, t] c}\right)$.

We can write Itô's formula for $e^{-r \lambda} V_{\lambda, t}$ since, for fixed $t$, the process $\left(V_{\lambda, t}\right)_{\lambda \in[0, t]}$ is a

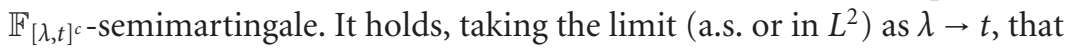

$$
\begin{aligned}
\tilde{V}_{t}= & \mathbb{E}\left(V_{0} / \mathbb{F}_{t^{c}}\right)+\int_{0}^{t} e^{-r s} h_{s} r e^{r s} d s-\int_{0}^{t} e^{-r s} H_{s} \mathbb{E}\left(D_{s} S_{0} / \mathbb{F}_{[s, t]^{c}}\right) d W_{s} \\
& +\int_{0}^{t} e^{-r s} \sigma H_{s} \mathbb{E}\left(S_{s} / \mathbb{F}_{[s, t]^{c}}\right) d W_{s}+\int_{0}^{t} e^{-r s} b H_{s} \mathbb{E}\left(S_{s} / \mathbb{F}_{[s, t]^{c}}\right) d s+\int_{0}^{t} V_{s, t}\left(-r e^{-r s}\right) d s \\
= & \mathbb{E}\left(V_{0} / \mathbb{F}_{t^{c}}\right)-\int_{0}^{t} e^{-r s} H_{s} \mathbb{E}\left(D_{s} S_{0} / \mathbb{F}_{[s, t]^{c}}\right) d W_{s} \\
& +\int_{0}^{t} e^{-r s} \sigma H_{s} \mathbb{E}\left(S_{s} / \mathbb{F}_{[s, t]^{c}}\right) d W_{s}+\int_{0}^{t} e^{-r s}(b-r) H_{s} \mathbb{E}\left(S_{s} / \mathbb{F}_{[s, t]^{c}}\right) d s .
\end{aligned}
$$

On the other hand, writing Itô's formula for $e^{-r \lambda} S_{\lambda, t}$ with

$$
S_{\lambda, t}=\mathbb{E}\left(S_{0} / \mathbb{F}_{t^{c}}\right)+\int_{0}^{\lambda} \sigma \mathbb{E}\left(S_{s} / \mathbb{F}_{[s, t]^{c}}\right) d W_{s}+\int_{0}^{\lambda} b \mathbb{E}\left(S_{s} / \mathbb{F}_{[s, t]^{c}}\right) d s=\mathbb{E}\left(S_{\lambda} / \mathbb{F}_{[\lambda, t]^{c}}\right),
$$

we get

$$
\tilde{S}_{t}=\mathbb{E}\left(S_{0} / \mathbb{F}_{t^{c}}\right)+\int_{0}^{t} e^{-r s} \sigma \mathbb{E}\left(S_{s} / \mathbb{F}_{[s, t]^{c}}\right) d W_{s}+\int_{0}^{t} e^{-r s}(b-r) \mathbb{E}\left(S_{s} / \mathbb{F}_{[s, t]^{c}}\right) d s .
$$

Identity (4.7) follows from (4.9) and the above equation using Definition 2.1. The proof of the necessary part is not more difficult.

Let $T$ be the exercise time. In the classical Black-Scholes settings, to prove the nonexistence of arbitrage, it suffices to exhibit a probability measure equivalent to $P$ under which the discounted price $\tilde{S}$ is a martingale. In our case, we have the following.

Proposition 4.3. The unique probability measure $\tilde{P}$ equivalent to $P$ under which the process $\tilde{S}_{t} / E\left(S_{0} / \mathbb{F}_{t^{c}}\right)$ is a martingale is given by the Radon-Nikodym derivative

$$
\frac{d \tilde{P}}{d P}=\exp \frac{r-\mu}{\sigma} W_{T}-\frac{1}{2} \frac{(r-\mu)^{2}}{\sigma^{2}} T \quad P \text {-a.s. }
$$

Under probability $\tilde{P}$, the process $\tilde{W}_{t}=W_{t}+((b-r) / \sigma) t$ is a standard Brownian motion and the discounted price $\tilde{S}$ satisfies the equation

$$
\tilde{S}_{t}=\mathbb{E}\left(\tilde{S}_{0} / \mathbb{F}_{t^{c}}\right)+\int_{0}^{t} \sigma \mathbb{E}\left(\tilde{S}_{s} / \mathbb{F}_{[s, t]^{c}}\right) d \tilde{W}_{s}
$$


Proof. Denote $Z_{t}=\tilde{S}_{t} / E\left(S_{0} / \mathbb{F}_{t^{c}}\right)=e^{-r t} e^{\sigma W_{t}+\left(b-\sigma^{2} / 2\right) t}$. It is well known that there exist the unique probability $\tilde{P}$ and the Brownian motion $\tilde{W}$ as above and it holds that $Z_{t}=1+$ $\int_{0}^{t} \sigma Z_{s} d \tilde{W}_{s}$. Taking into account that the natural filtrations of $W$ and $\tilde{W}$ coincide, we get

$$
\tilde{S}_{t}=\mathbb{E}\left(\tilde{S}_{0} / \mathbb{F}_{t^{c}}\right) Z_{t}=\mathbb{E}\left(\tilde{S}_{0} / \mathbb{F}_{t^{c}}\right)+\int_{0}^{t} \sigma \mathbb{E}\left(\tilde{S}_{0} / \mathbb{F}_{t^{c}}\right) Z_{s} d \tilde{W}_{s}=\mathbb{E}\left(\tilde{S}_{0} / \mathbb{F}_{t^{c}}\right)+\int_{0}^{t} \sigma \mathbb{E}\left(\tilde{S}_{s} / \mathbb{F}_{[s, t] c}\right) d \tilde{W}_{s}
$$

Remark 4.4. Note that, by Corollary 3.3, we have $\tilde{S}_{t}=\mathbb{E}\left(\tilde{S}_{0} / \mathbb{F}_{t^{c}}\right) e^{\sigma \tilde{W}_{t}-\left(\sigma^{2} / 2\right) t}$. Also, an immediate consequence of Propositions 4.2 and 4.3 is the fact that the market is complete and has no arbitrage opportunities.

Consider $V_{T}=\left(S_{T}-K\right)^{+}$the payoff function of the European call option with exercise time $T$ and strike price $K$. Denote by $\tilde{E}$ the expectation with respect to $\tilde{P}$ and by $\tilde{D}$ the Malliavin derivative with respect to $\tilde{W}$. By formulas (4.7) and (4.13), we have

$$
\left.\tilde{V}_{t}=\tilde{E}\left(V_{0} / \mathbb{F}_{t^{c}}\right)-\int_{0}^{t} H_{s} \tilde{\mathbb{E}}\left(\tilde{D}_{s} S_{0} / \mathbb{F}_{[s, t]^{c}}\right) d \tilde{W}_{s}+\int_{0}^{t} \sigma H_{s} \tilde{\mathbb{E}}_{\left(\tilde{S}_{s} / \mathbb{E}\right.}[s, t]^{c}\right) d \tilde{W}_{s}
$$

Taking the conditional expectation with respect to the $\sigma$-algebra $\mathbb{F}_{t}$, we obtain

$$
\left.\tilde{\mathbb{E}}\left(\tilde{V}_{t} / \mathbb{F}_{t}\right)=\tilde{\mathbb{E}}\left(V_{0}\right)-\int_{0}^{t} H_{s} \tilde{\mathbb{E}}\left(\tilde{D}_{s} S_{0} / \mathbb{F}_{s}\right) d \tilde{W}_{s}+\int_{0}^{t} \sigma H_{s} \tilde{\mathbb{E}}_{\left(S_{s}\right.} / \mathbb{F}_{s}\right) d \tilde{W}_{s}
$$

Therefore, the process $\left(\tilde{\mathbb{E}}\left(\tilde{V}_{t} / \mathbb{F}_{t}\right)\right)_{t \in[0,1]}$ is a martingale and, for every $t \leq T$, it holds that $\tilde{\mathbb{E}}\left(\tilde{V}_{t} / \mathbb{F}_{t}\right)=\tilde{\mathbb{E}}\left(\tilde{V}_{T} / \mathbb{F}_{t}\right)$ or

$$
\tilde{\mathbb{E}}\left(V_{t} / \mathbb{F}_{t}\right)=\tilde{\mathbb{E}}\left(e^{-r(T-t)} V_{T} / \mathbb{F}_{t}\right)
$$

We have the following option valuation Black-Scholes formula.

Proposition 4.5. Assume that the terminal value is given by $V_{T}=f\left(S_{T}\right)$ with $f(x)=$ $(x-K)^{+}$and the initial price of the risky asset is $S_{0}=W_{1}+c$, where $c$ is a positive constant. Then

$$
\tilde{\mathbb{E}}\left(V_{t} / \mathbb{F}_{t}\right)=G\left(t, \frac{S_{t}}{W_{1}-W_{t}+c}\right)
$$

where

$$
\begin{aligned}
G(t, x)= & \frac{1}{\sqrt{2 \pi(1-T)}} \\
& \times\left[x\left(\int_{\mathbb{R}} e^{-u^{2} /(1-T)}(u+c) N\left(d_{1}\right) d u\right)-K e^{-r(T-t)} \int_{\mathbb{R}} e^{-u^{2} /(1-T)} N\left(d_{2}\right) d u\right]
\end{aligned}
$$


with

$$
\begin{aligned}
& d_{1}=d_{1}(x, u)=\frac{\ln (K / x(u+c))+\left(r+\sigma^{2} / 2\right)(T-t)}{\sigma \sqrt{T-t}} \\
& d_{2}=d_{2}(x, u)=d_{1}-\sigma \sqrt{T-t}
\end{aligned}
$$

and $N(d)=(1 / \sqrt{2 \pi}) \int_{-\infty}^{d} e^{-x^{2} / 2} d x$.

Proof. Using the fact that the increments of the Wiener process are independent on disjoint intervals, the Markov property and (4.17) imply that

$$
\begin{aligned}
\tilde{\mathbb{E}}\left(V_{t} / \mathbb{F}_{t}\right) & =\tilde{\mathbb{E}}\left[e^{-r(T-t)} f\left(S_{T}\right) / \mathbb{F}_{t}\right] \\
& =\tilde{\mathbb{E}}\left[e^{-r(T-t)} f\left(e^{\sigma W_{t}+\left(r-\sigma^{2} / 2\right) t} \tilde{\mathbb{E}}\left(S_{0} / \mathbb{F}_{T^{c}}\right) e^{\sigma\left(W_{T}-W_{t}\right)+\left(r-\sigma^{2} / 2\right)(T-t)}\right)\right] \\
& =G\left(t, \frac{S_{t}}{\tilde{\mathbb{E}}\left(S_{0} / \mathbb{F}_{t^{c}}\right)}\right),
\end{aligned}
$$

where

$$
G(t, x)=e^{-r(T-t)} \tilde{\mathbb{E}}\left[f\left(x \mathbb{E}\left(S_{0} / \mathbb{F}_{T^{c}}\right) e^{\sigma\left(W_{T}-W_{t}\right)+\left(r-\sigma^{2} / 2\right)(T-t)}\right)\right] .
$$

Since $\mathbb{E}\left(S_{0} / \mathbb{F}_{T^{c}}\right)=W_{1}-W_{T}+c$, by the joint normal distribution of $\left(W_{1}-W_{T}, W_{T}-W_{t}\right)$,

$$
\begin{aligned}
G(t, x)= & \frac{1}{\sqrt{2 \pi(1-T)}} \\
& \times \int_{\mathbb{R}} e^{-u^{2} / 2(1-T)}\left(\frac{e^{-r(T-t)}}{\sqrt{2 \pi(T-t)}} \int_{\mathbb{R}} f\left(x(u+c) e^{\sigma v+\left(r-\sigma^{2} / 2\right)(T-t)}\right) e^{-v^{2} / 2(T-t)} d v\right) d u .
\end{aligned}
$$

We refer to classical arguments (see [5]) to get

$$
\begin{aligned}
\frac{e^{-r(T-t)}}{\sqrt{2 \pi(T-t)}} \int_{\mathbb{R}} f\left(x(u+c) e^{\sigma v+\left(r-\sigma^{2} / 2\right)(T-t)}\right) e^{-v^{2} / 2(T-t)} d v \\
=x(u+c) N\left(d_{1}(x, u)\right)-K e^{-r(T-t)} N\left(d_{2}(x, u)\right),
\end{aligned}
$$

where $d_{1}, d_{2}$ are given by (4.20) and the conclusion follows.

Since the market is complete, every bounded contingent claim is attainable. Therefore, it is of importance to find the expression of the replicant portfolio. This is given in Proposition 4.6.

Proposition 4.6. Under the hypothesis of Proposition 4.5 and denoting $g(t, x)=$ $e^{-r t} G\left(t, e^{r t} x\right)$, the replicant portfolio is given by

$$
\begin{aligned}
& H_{t}=\left(c \sigma \frac{\tilde{S}_{t}}{\tilde{\mathbb{E}}\left(S_{0} / \mathbb{F}_{t^{c}}\right)}-1\right)^{-1} \sigma \frac{\partial g}{\partial x}\left(t, \frac{\tilde{S}_{t}}{\tilde{\mathbb{E}}\left(S_{0} / \mathbb{F}_{t^{c}}\right)}\right), \\
& h_{t}=G\left(t, \frac{S_{t}}{W_{1}-W_{t}+c}\right)-c H_{t} \frac{\tilde{S}_{t}}{\tilde{\mathbb{E}}\left(S_{0} / \mathbb{F}_{t^{c}}\right)} .
\end{aligned}
$$


Proof. Denote $M_{t}=\tilde{S}_{t} / \tilde{\mathbb{E}}_{(}\left(S_{0} / \mathbb{F}_{t^{c}}\right)$. We utilize the classical procedure to determine the unknown quantities $h$ and $H$. We have that

$$
\begin{aligned}
\tilde{\mathbb{E}}\left(\tilde{V}_{t} / \mathbb{F}_{t}\right) & =e^{-r t} \tilde{\mathbb{E}}\left(V_{t} / \mathbb{F}_{t}\right)=e^{-r t} G\left(t, \frac{S_{t}}{\tilde{\mathbb{E}}\left(S_{0} / \mathbb{F}_{t^{c}}\right)}\right) \\
& =e^{-r t} G\left(t, e^{r t} \frac{\tilde{S}_{t}}{\tilde{\mathbb{E}}\left(S_{0} / \mathbb{F}_{t^{c}}\right)}\right)=e^{-r t} G\left(t, e^{r t} M_{t}\right)
\end{aligned}
$$

with $G$ a $C^{\infty}$ function on $[0, T) \times \mathbb{R}$. Writing Itô's formula for $g\left(t, M_{t}\right)$, we obtain

$$
\begin{aligned}
g\left(t, M_{t}\right)= & g\left(0, M_{0}\right)+\int_{0}^{t} \sigma \frac{\partial g}{\partial x}\left(u, M_{u}\right) M_{u} d \tilde{W}_{u} \\
& +\int_{0}^{t} \frac{\partial g}{\partial t}\left(u, M_{u}\right) d u+\frac{1}{2} \int_{0}^{t} \frac{\partial^{2} g}{\partial x^{2}}\left(u, M_{u}\right) \sigma^{2} M_{u}^{2} d u
\end{aligned}
$$

Note first that the bounded variation part is zero. On the other hand, by (4.7),

$$
\tilde{\mathbb{E}}\left(\tilde{V}_{t} / \mathbb{F}_{t}\right)=\tilde{\mathbb{E}}\left(V_{0}\right)-\int_{0}^{t} H_{s} E\left(\tilde{D}_{s} S_{0} / \mathbb{F}_{s}\right)+\int_{0}^{t} \sigma H_{s} \tilde{\mathbb{E}}\left(\tilde{S}_{s} / \mathbb{F}_{s}\right) d \tilde{W}_{s}
$$

By (4.27) and (4.28), the natural candidate for $H$ satisfies $\sigma(\partial g / \partial x)\left(s, M_{s}\right) M_{s}=$ $\left.\sigma H_{s} \tilde{\mathbb{E}}_{\left(\tilde{S}_{s}\right.} / \mathbb{F}_{s}\right)$ and since $\tilde{\mathbb{E}}\left(\tilde{S}_{s} / \mathbb{F}_{s}\right)=\tilde{\mathbb{E}}\left(S_{0}\right) M_{s}$, we obtain relation $(4.25)$.

\section{References}

[1] R. Buckdahn, Linear Skorohod stochastic differential equations, Probab. Theory Related Fields 90 (1991), no. 2, 223-240.

[2] Skorohod stochastic differential equations of diffusion type, Probab. Theory Related Fields 93 (1992), no. 3, 297-323.

[3] R. Buckdahn and D. Nualart, Linear stochastic differential equations and Wick products, Probab. Theory Related Fields 99 (1994), no. 4, 501-526.

[4] P. E. Kloeden and E. Platen, Numerical Solution of Stochastic Differential Equations, Applications of Mathematics, vol. 23, Springer-Verlag, Berlin, 1992.

[5] D. Lamberton and B. Lapeyre, Introduction au Calcul Stochastique Appliqué à la Finance [Introduction to Stochastic Calculus Applied to Finance], Edition Ellipses, Paris, 1991.

[6] D. Nualart, The Malliavin Calculus and Related Topics, Probability and Its Applications (New York), Springer-Verlag, New York, 1995.

[7] D. Nualart and É. Pardoux, Stochastic calculus with anticipating integrands, Probab. Theory Related Fields 78 (1988), no. 4, 535-581.

[8] A. V. Skorohod, On a generalization of a stochastic integral, Teor. Verojatnost. i Primenen 20 (1975), 219-233.

[9] C. A. Tudor, Martingale-type stochastic calculus for anticipating integral processes, Bernoulli 10 (2004), no. 2, 313-325.

Ciprian A. Tudor: Laboratoire de Probabilités et Modèles Aléatoires, Université de Paris 6, 4 Place Jussieu, 75252 Paris Cedex 5, France

E-mail address: tudor@ccr.jussieu.fr 


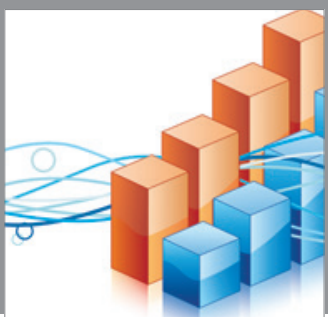

Advances in

Operations Research

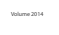

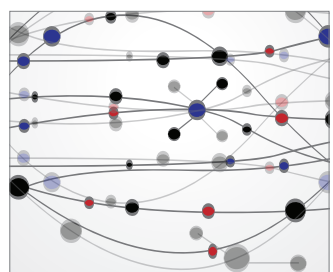

\section{The Scientific} World Journal
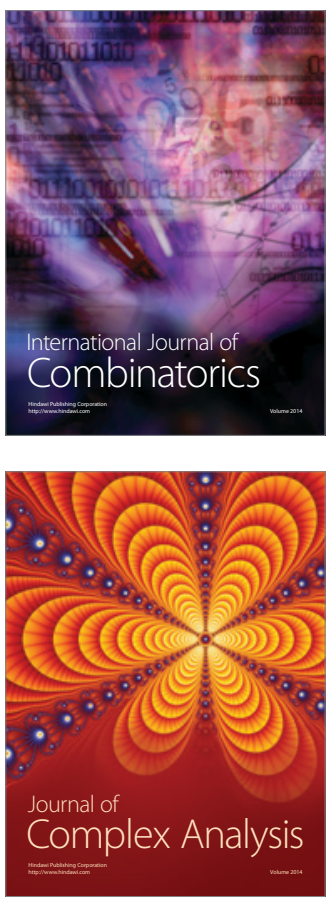

International Journal of

Mathematics and

Mathematical

Sciences
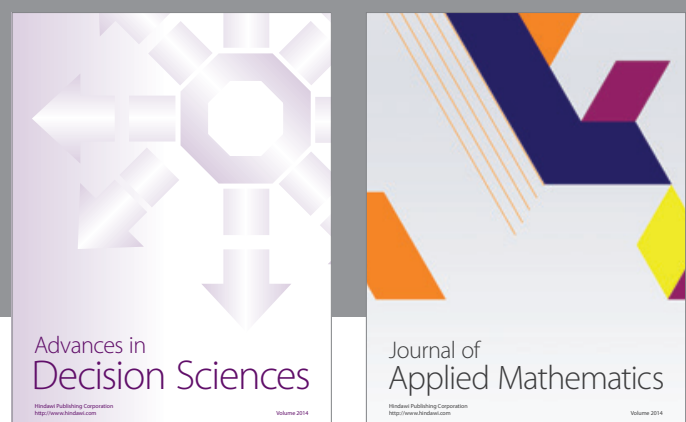

Journal of

Applied Mathematics
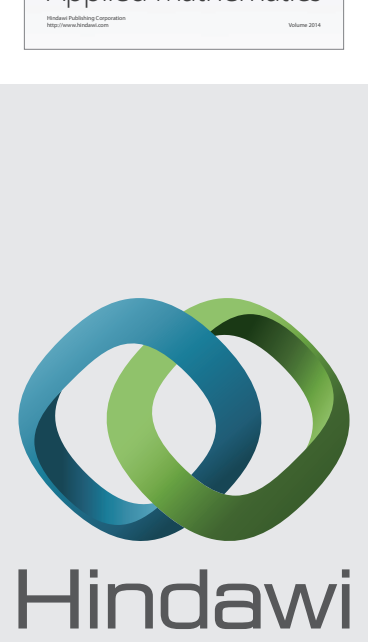

Submit your manuscripts at http://www.hindawi.com
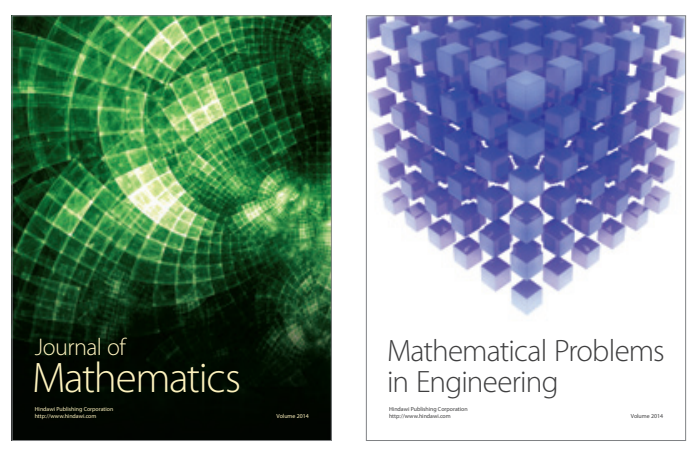

Mathematical Problems in Engineering
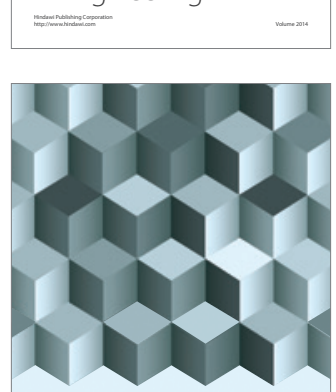

Journal of

Function Spaces
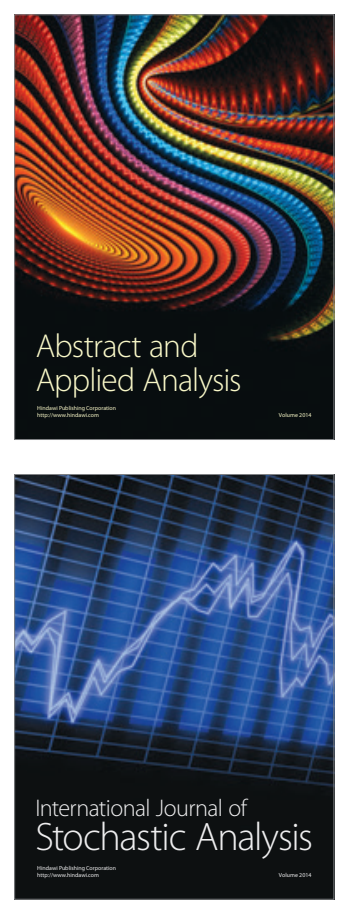

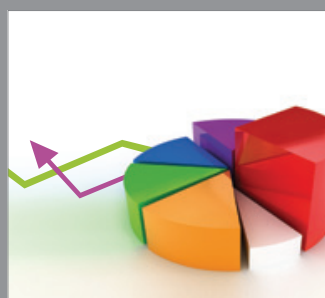

ournal of

Probability and Statistics

Promensencen
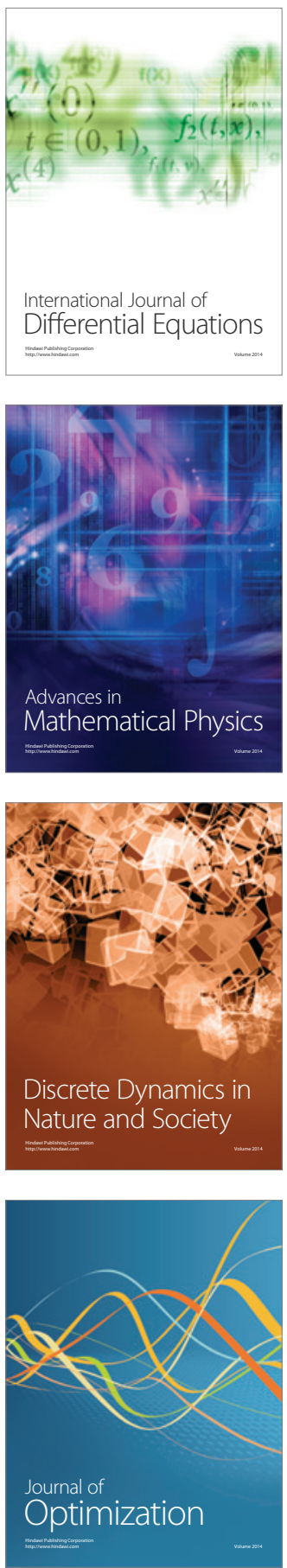